\title{
Contract Theory Based Cooperative Spectrum Sharing with J oint Power and Bandwidth Optimization
}

\author{
Weidang Lu ${ }^{1,2}$, Chenxin $\mathrm{He}^{1}$, Yuanrong Lin ${ }^{1}$, Hong Peng ${ }^{1,2}$, Xin Liu ${ }^{3}$ \\ ${ }^{1}$ College of Information Engineering, Zhejiang University of Technology \\ Hangzhou, Zhejiang 310023 - China \\ [e-mail: \{luweid, ph\}@zjut.edu.cn] \\ ${ }^{2}$ Zhejiang Provincial Key Laboratory of Communication Networks and Applications, Zhejiang University of \\ Technology, Hangzhou, Zhejiang 310023 - China \\ ${ }^{3}$ School of Electronic Information and Electrical Engineering, Dalian University of Technology \\ Dalian, Liaoning 116024 - China \\ [e-mail: liuxinstar1984@dlut.edu.cn] \\ *Corresponding author: Weidang Lu
}

Received March 29, 2017; revised July 17, 2017; revised August 9, 2017; accepted August 20, 2017; Published December 31, 2017

\begin{abstract}
In this paper, we proposed a contract theory based cooperative spectrum sharing scheme with joint power and bandwidth optimization under asymmetric information, where the primary user (PU) does not know the secondary users' (SUs) private information. To improve performance, PU needs to provide incentives to stimulate nearby SUs to help forward its signal. By using contract theory, PU and SUs' negotiations are modeled as a labor market. PU and SUs act as the employer and employees, respectively. Specifically, SUs provide labor (i.e. the relay power, which can be used for forwarding PU's signal) in exchange for the reward (i.e. the spectrum access bandwidth which can be used for transmitting their own signals). PU needs to overcome a challenge how to balance the relationship between contributions and incentives for the SUs. We study the optimal contract design which consists of relay power and spectrum access bandwidth allocation. We show that the most efficient SUs will be hired by the PU to attend the cooperative communication. PU can achieve the same maximum utility as in the symmetric information scenario. Simulation results confirm that the utility of PU is significantly enhanced with our proposed cooperative spectrum sharing scheme.
\end{abstract}

Keywords: spectrum sharing; contract theory; resources allocation; decode-and-forward relaying

This work was supported by China National Science Foundation under Grand No. 61402416 and 61601221, Project funded by China Postdoctoral Science Foundation under Grand No. 2017M612027 


\section{Introduction}

The vision of improving the spectrum utilization and alleviating the scarcity of spectrum resources by using cognitive radio technology has led to a great deal of research. Due to the limitation of time and location, traditional fixed spectrum allocation mechanism is not efficient to meet the growing demand of wireless services. The design of cognitive radio networks is based on the concept of dynamic spectrum sharing where cognitive users can intelligently access the spectrum through spectrum sensing [1]. To make the spectrum sensing more efficient for the crowd sensor network, a generalized modeling approach for sensing data with an arbitrary abnormal component is proposed [2]. [3] gives an overview of applying various Kernel-based learning methods in spectrum sensing to solve both long-standing and emerging design problems.

In cooperative communication, system performance can be improved by obtaining diversity gain [4]. Integrating cooperative communication into cognitive radio network can effectively improve the spectrum utilization [5]. In cooperative spectrum sharing schemes, SUs can help forward PU's signal either by amplify-and-forward (AF) or decode-and-forward (DF) relay protocols [6,7]. [8] and [9] study cooperative spectrum sharing in the future 5G networks. Spectrum sensing and relay selection are studied in multiple-relay cognitive radio networks $[10,11]$. However, SUs are selfish and rational which leads them reluctant to take participate in PU's cooperative communication without incentives. PU needs to provide some compensation to encourage the SUs to participate in the cooperation.

There are three main compensation mechanisms, which are based on resource-exchange, money-exchange and reputation-exchange, respectively [12]. In resource-exchange mechanism, PU provides its resource (i.e., spectrum accessing time) to SUs, which can be used to transmit SUs' signals to exchange SUs to relay PU's signal [13-15]. In [13], the system is modeled as a non-cooperative game process, in which a unique point is obtained under the frame of Stackelberg equilibrium. [14] proposes a cooperative spectrum sharing strategy which optimizes the sum-rate of the SUs while ensuring the quality-of-service of PUs by sharing PUs' subchannels in OFDMA-based cognitive radio network. An improved cooperative resource sharing strategy considering the joint determination is proposed in [15]. Money-exchange mechanism has two different forms, namely, pricing and auction. Pricing is widely used when PUs know the value of resources and sell them via virtual currency or tokens. SUs have the right to determine whether to access PUs' spectrum according to the pricing provided by PUs $[16,17]$. In auction based mechanism, PUs and SUs make trading through submitting demand and bids to auctioneer, respectively, to do the two-way selection [18-20]. The above two compensation mechanisms are only suitable for the short-term memoryless systems, while the reputation-exchange mechanism can be used for the long-term systems, which focus on the long-term performance [21].

The above three compensation mechanisms all assume that PUs can obtain SUs' complete information such as channel conditions, resource requirements, and costs for cooperation transmission, which is unrealristic for practical implements. Contract theory is an effective method to design incentive compatible mechanisms in a monopoly market under asymmetric information [22]. [23] studies a monopolist-dominated quality-price contract design to maximize PU's utility. The contract contains a set of quality and price combinations for the sold spectrum, which is designed for each SU's type. A contract-theory based framework under asymmetric channel information is proposed in [24], in which the contract comprises a set of targeted SNR at PR and credit items. Contract design including spectrum access time 
and power is proposed in [25], where one PU designs and broadcasts the designed contract, if SUs accept PU's contract, they forward the PU's signal by using a fraction of their power and gain spectrum access time.

In practical, if too many SUs accept PU's contract, it will be difficult for all these SUs to access to the very small pieces of time divided from one time slot. Therefore, in this paper, we propose a contract design including spectrum access bandwidth and relay power. Specifically, the selected SUs provide the relay power to help forward PU's signal in exchange for the dedicated spectrum access bandwidth to transmit their own signals.

The main contributions of the paper are summarized as follows: First, we propose optimal contract design under symmetric and asymmetric information, respectively. We show that the optimal cotract with asymmetric information achieves the same maximum PU utility as in the symmetric information scenario. Second, we show that under asymmetric information, the optimal contract is feasible if and only if it is incentive compatible (IC) and individually rational (IR) for each SU. The necessary and sufficient conditions are characterized for a contract to be IC and IR systematically. Finally, simulation results demonstrate that PU's utility is significantly enhanced with our proposed strategy.

The left of this paper is organized as follows. Section 2 presents the system model and formulates the problem. In section 3, we show the optimal contract design under symmetric and asymmetric information. Computer simulation results are demonstrated in Section 4 to illustrate the performance of the proposed strategy. Finally we make a conclusion in Section 5.

\section{System Model and Problem Formulation}

\subsection{System model}

We consider a typical cooperative cognitive radio network composed of one PU and multiple SUs as shown in Fig. 1. PU comprises a transmitter PT and a receiver PR. Each SU has a dedicated transmitter $S T$ and receiver $S R$. The PU has the exclusive frequency band. However, due to path loss, shadowing, or interference, $P T \rightarrow P R$ link experiences a poor link quality. On the other hand, SUs surrounding the PU want to access the licensed spectrum bands and transmit their own signals, and they cannot transmit without the PU's permission. The PU can hire nearby SUs to relay its signal, while providing dedicated spectrum access bandwidth to them which can be used to transmit SUs' signals.

As shown in Fig. 1, the negotiation between PU and SUs before transmission includes the following three steps:

- Step 1. PU broadcasts a testing signal, which contains the contract items (relay power and dedicated spectrum access bandwidth), to PR and SUs.

- Step 2. The $S U_{k}$ which can decode PU's signal correctly feedback a signal to the PU. These $S U_{k}$ will be selected into the candidate access set denoted as $\Omega=\{1,2, \ldots, K\}$.

- Step 3. PU selects a proper subset of SUs to start the cooperative communication.

The third step can be regardes as cooperative communication progress. As shown in Fig. 2, it is divided into the following two equal phases:

- In the first phase, PT transmits the signal to PR through the direct transmission link, while $S U_{k}(k \in \Omega)$ also receives PU's signal.

- In the second phase, each $S U_{k}(k \in \Omega)$ forwards PU's signal by using a fraction of 
power and the licensed spectrum bandwidth, and uses the remained bandwidth rewarded by PU and power to transmit own signals to $S R_{k}$.
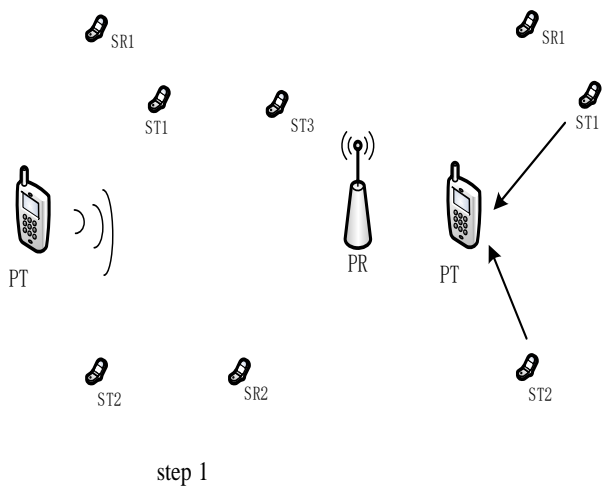

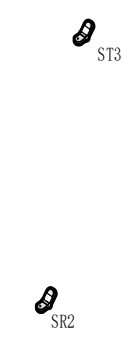

step 2
$\boldsymbol{\otimes}_{\mathrm{SR} 1}$

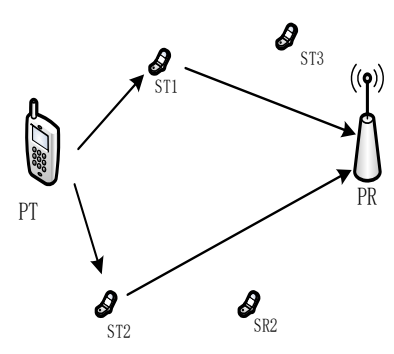

step 3

Fig. 1. Negotiation steps

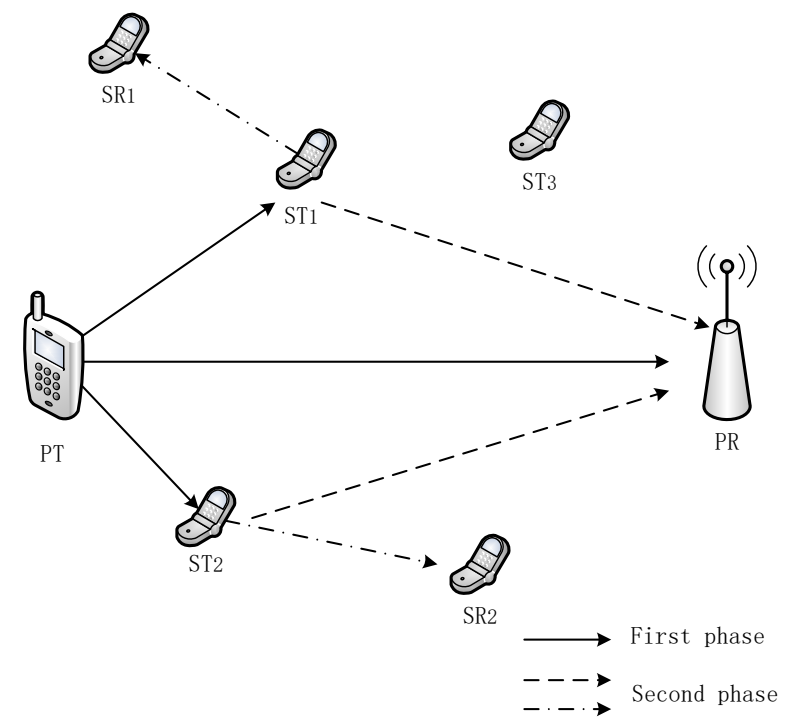

Fig. 2. Cooperative communication progress

PU and SUs have conflicting objectives in the negotiation. The PU wants to increase its transmission rate, which requires SUs to use more power and bandwidth to relay PU's signal. However, it will reduce SUs' performance. And $S U_{k}$ wants to increase its own performance by obtaining more dedicated bandwidth $b_{k}$, which will reduce PU's transmission rate. In Sections 2.2, we will explain in details how the PU and SUs evaluate the tradeoff between relay power and bandwidth allocations. In Section 3, we propose a contract-based framework which brings the PU and SUs together and resolves the conflicts.

\subsection{Problem formulation}

\section{a) PU model}

We first calculate PU's transmission rate when PU transmits its signal through the direct link without any SUs spectrum sharing. The PU's achievable rate can be written as: 


$$
R_{d}=\log \left(1+S N R_{P T, P R}\right)
$$

where $S N R_{P T, P R}$ denotes the signal-to-noise ratio between PT to PR.

PU will seek help from the nearby SUs to improve its performance, when $P T \rightarrow P R$ link experiences a poor link quality. The selected SUs decode the received signal and help forward it to PR with the following two phases transmission.

In the first transmission phase, as shown in Fig.2, PT broadcasts its signal to PR and the selected $S T_{k}, k \in \Omega$. Thus, the achievable rate of $P T \rightarrow P R$ link can be written as:

$$
R_{1}=\frac{1}{2} \log \left(1+S N R_{P T, P R}\right)
$$

where constant $1 / 2$ is due to the two half phases transmission.

In the second phase, the selected $S T_{k}, k \in \Omega$ decode PU's signal and allocate a fraction of licensed spectrum band to help PT forward its signal to PR. The total achievable rate of PU with two phases transmission can be written as [26]:

$$
\begin{aligned}
R_{\text {tot }} & =\frac{1}{2} R_{d}+\frac{1}{2}\left(1-\sum_{k \in \Omega} b_{k}\right) \log \left(1+\sum_{k \in \Omega} S N R_{S T_{k}, P R}\right) \\
& =\frac{1}{2} R_{d}+\frac{1}{2}\left(1-\sum_{k \in \Omega} b_{k}\right) \log \left(1+\frac{\sum_{k \in \Omega} p_{k}}{n_{0}}\right)
\end{aligned}
$$

where $n_{0}$ denotes the noise power, $b_{k}$ and $p_{k}$ denote the dedicated spectrum access bandwidth allocated to $S T_{k}$ and received power at PR from $S T_{k}$, respectively. For simplicity, it is assumed that the noise at all the nodes is addictive white Gaussian noises (AWGN).

The PU's objective is to maximize its utility (i.e., the total transmission rate through two phases transmission) as follows [25]:

$$
U_{P U}=R_{\text {tot }}=\frac{1}{2} R_{d}+\frac{1}{2}\left(1-\sum_{k \in \Omega} b_{k}\right) \log \left(1+\frac{\sum_{k \in \Omega} p_{k}}{n_{0}}\right)
$$

Definition 1 (Performance Constraint). The PU's utility with cooperative transmission should be larger than its utility with direct transmission $U_{0}=R_{d}$, i.e.,

$$
U_{P U} \geq U_{0}
$$

It is remarkable that PU will only choose to use cooperative communiation if the utility $U_{P U}$ is larger than $U_{0}$, which also means that SUs can only access to the PU's spectrum only when they can help improve PU's utility. In the following analysis, we assume that the direct transmission rate is small such that PU is willing to seek cooperation.

\section{b) SU model}

Next, we discuss how SUs evaluate bandwidth allocation $b_{k}$ and relay power $p_{k}$. Note that the channel coefficient of $S T_{k}$ to PR link is different (i.e. $h_{k}$ is different). And each selected $S T_{k}$ has different transmission rate requirement (i.e., $R_{k}$ ) and transmission power (i.e. $p_{s k}$ ) and received power at SR $p_{s}=p_{s k} / h_{S T, S R}$. Each SU has own unit power consumption cost (i.e. $C_{k}$ ). We can find that each SU may have different personal information. And these personal information are only known by SU itself. 
We define $\pi_{k}$ as the payoff of $S U_{k}$, which can be written as:

$$
\begin{aligned}
\pi_{k} & =b_{k} R_{k}-\left(b_{k} p_{s k}+\frac{1}{2} p_{r k}\right) C_{k} \\
& =b_{k} R_{k}-\left(b_{k} p_{s k}+\frac{1}{2} \frac{p_{k}}{h_{k}}\right) C_{k}
\end{aligned}
$$

where $p_{r k}$ is the relay power of the transmitter $S T_{k}$ and $p_{r k}=\frac{p_{k}}{h_{k}}$. Multiplying both sides by $\frac{2 h_{k}}{C_{k}}$, we can obtain a normalized payoff written as [25]:

$$
\tilde{\pi}_{k}=\frac{2 h_{k}\left(R_{k}-C_{k} p_{s k}\right)}{C_{k}} b_{k}-p_{k}
$$

According to SU's different personal information, SUs can be classified into different types. Each $S U_{k}$ has a unique type $\theta_{k}$, which can be defined as:

$$
\theta_{k}=\frac{2 h_{k}\left(R_{k}-C_{k} p_{s k}\right)}{C_{k}}>0
$$

We can find that a larger type- $\theta_{k}$ implies the SU has better channel quality on the link of $S T \rightarrow P R$, lower cost of power consumption or better transmission performance.

Thus, the payoff of $S U_{k}$ can be written as:

$$
\tilde{\pi}\left(p_{k}, b_{k}\right)=\theta_{k} b_{k}-p_{k}
$$

\section{Contract Formulation}

As mentioned in the previous section, there is a conflict of objectives between PU and SUs. The PU expects that SUs can use more relay power and bandwidth to forward its signal in order to achieve larger the utility, while SUs always want to increase their own performance by obtaining more dedicated bandwidth $b_{k}$. We introduce a contract incentive mechanism to resolve the above conflicting objectives.

Note that SUs do not have the obligation of consuming their own energy to help improve PU's performance without rewards. Thus, the PU needs to design a contract to attract the SUs to forward PU's signal. The most important question is how to design an optimal contract which balances the incentives and contributions.

We consider SUs have $K$ differdent types. The set of types are denoted by a set $\Theta=\left\{\theta_{1}, \theta_{2}, . ., \theta_{K}\right\}$. Without loss of generality, assume $\theta_{1}<\theta_{2}<\ldots<\theta_{K}$. The feasible contract contains $K$ power-bandwidth items, i.e., relay power received at PR and spectrum access bandwidth, which can be denoted as

$$
C=\left\{\left(p\left(\theta_{k}\right), b\left(\theta_{k}\right), \theta_{k} \in \Theta\right\}\right.
$$


It can be simplified as

$$
C=\left\{\left(p_{k}, b_{k}, k \in \Omega\right\}\right.
$$

Once the contract $\left(p_{k}, b_{k}\right)$ is accept by $S U_{k}$, which means that $S U_{k}$ should forward PU's signal with the relay power received at PR of $p_{k}$, and could obtain the spectrum access bandwidth of $b_{k}$ to tarnsmit its own signal in return.

\subsection{Optimal contract design under symmetric information}

Under the symmetric information scenario, PU knows the precise type of each SU. Then, PU has the ability to monitor and ensure that SUs only accept the corresponding contract pairs designed for them. Considering that SUs are selfish and rational, PU needs to ensure that SUs can get non-negative rewards. That means the contract pairs need to satisfy the individual rationality constraint formulated as follows.

Definition 2 (Individual Rationality, IR). A contract satisfies the IR constraint if and only if each type- $\theta_{k}$ SU obtains nonnegative payoff when it chooses the contract term designed for its type, i.e.,

$$
\theta_{k} b_{k}-p_{k} \geq 0, \quad \forall k \in \Omega
$$

The optimal contract is designed to maximize PU's utility, which can be written as:

$$
\max _{\left\{\left(p_{k}, b_{k}\right), \forall k \in \Omega\right\}} \frac{1}{2} R_{d}+\frac{1}{2}\left(1-\sum_{k \in \Omega} b_{k}\right) \log \left(1+\frac{\sum_{k \in \Omega} p_{k}}{n_{0}}\right)
$$

subject to

$$
\left\{\begin{array}{l}
\theta_{k} b_{k}-p_{k} \geq 0, \quad \forall k \in \Omega \\
\frac{1}{2} R_{d}+\frac{1}{2}\left(1-\sum_{k \in \Omega} b_{k}\right) \log \left(1+\frac{\sum_{k \in \Omega} p_{k}}{n_{0}}\right) \geq R_{d}
\end{array}\right.
$$

Lemma1. In an optimal contract, the selected $S U_{k}$ obtains zero payoff by accepting the corresponding contract item ${ }^{1}$, i.e.,

$$
\theta_{k} b_{k}-p_{k}=0, \quad \forall k \in \Omega
$$

Proof. According to IR constraint, we have $\theta_{k} b_{k}-p_{k} \geq 0, \forall k \in \Omega$. Assuming that there exists an optimal contract item satisfying the condition $\theta_{k} b_{k}-p_{k}>0$. PU can improve its utility by increasing $p_{k}$ until $p_{k}$ reaches $\theta_{k} b_{k}$, which results in maximum utility of PU, and thus complete the proof.

Theorem 1. PU prefers to hire the SU with highest type as cooperative relay in the optimal contract, which indicates that only the corresponding contract pair of the highest type is non-negative, and all others equal to zero. It can be denoted as: $\left(P_{K}, b_{K}\right)>0$ and $\left(P_{k}, b_{k}\right)=0, \forall k \in \Omega, k<K$.

Proof. Suppose that there exists an optimal contract with $b_{k}>0, k<K$. The total available bandwidth allocation is

\footnotetext{
${ }^{1}$ Although the payoff of the selected $S U_{k}$ is zero, from (8) and (14), we can find $R_{k}=\theta_{k} C_{k} /\left(2 h_{k}\right)+C_{k} P_{s k}$ which is not zero. It means $S U_{k}$ can gain the chance to transmit its own signal. Thus, $S U_{k}$ will benefit from the proposed spectrum sharing scheme.
} 


$$
B^{\prime}=\sum_{k \in \Omega} b_{k}
$$

Then PU's utility can be written as:

$$
U_{P U 1}=\frac{1}{2} R_{d}+\frac{1}{2}\left(1-B^{\prime}\right) \log \left(1+\sum_{k \in \Omega} \frac{\theta_{k} b_{k}}{n_{0}}\right)
$$

If we allocate all the $B^{\prime}$ to the highest type $\theta_{K}$, then $b_{K}=B^{\prime}$. PU's utility can be written as:

$$
U_{P U 2}=\frac{1}{2} R_{d}+\frac{1}{2}\left(1-B^{\prime}\right) \log \left(1+\frac{\theta_{K} b_{K}}{n_{0}}\right)
$$

According to the inequality zoom principle, it can be derived as:

$$
\sum_{k \in \Omega}\left(\frac{\theta_{k} b_{k}}{n_{0}}\right) \leq \sum_{k \in \Omega}\left(\frac{\theta_{K} b_{k}}{n_{0}}\right)=\frac{\theta_{K} \sum_{k \in \Omega} b_{k}}{n_{0}}=\frac{\theta_{K} B^{\prime}}{n_{0}}=\frac{\theta_{K} b_{K}}{n_{0}}
$$

Based on the above analysis, we can find $U_{P U 2} \geq U_{P U 1}$, which means that the SU with highest type can offer the largest help to the PU's utility under the condition of a given total available bandwidth allocation.

Using Lemma 1 and Theorem 1, the PU's utility maximization problem (14) can be simplified as:

$$
\max _{\left(b_{K} \geq 0\right)} \frac{1}{2} R_{d}+\frac{1}{2}\left(1-b_{K}\right) \log \left(1+\frac{\theta_{K} b_{K}}{n_{0}}\right)
$$

Take the first derivation of $U_{P U}$ with regard to $b_{K}$, we can obtain:

$$
\frac{d U_{P U}}{d b_{K}}=\frac{\theta_{K}\left(1-b_{K}\right)}{2\left(\theta_{K} b_{K}+n_{0}\right)}-\frac{1}{2} \log \left(1+\frac{\theta_{K} b_{K}}{n_{0}}\right)
$$

Then take the second derivation of $U_{P U}$ with $b_{K}$, we can obtain:

$$
\frac{d^{2} U_{P U}}{d b_{K}{ }^{2}}=-\frac{\theta_{K}\left(\theta_{K} b_{K}+\theta_{K}+2 n_{0}\right)}{2\left(\theta_{K} b_{K}+n_{0}\right)^{2}}
$$

We can find that (21) is a strictly concave function due to the second derivation of $U_{P U}(21)$ is always negative. Then, the optimal bandwidth allocation can be obtained when the first derivation of $U_{P U}$ equals to zero. Thus, the optimal bandwidth and power allocation can be written as:

$$
\begin{gathered}
b_{K}=\frac{\theta_{K}+n_{0}}{\theta_{K} * W\left(\frac{e^{*}\left(\theta_{K}+n_{0}\right)}{n_{0}}\right)}-\frac{n_{0}}{\theta_{K}} \\
p_{K}=\frac{\theta_{K}+n_{0}}{W\left(\frac{e^{*}\left(\theta_{K}+n_{0}\right)}{n_{0}}\right)}-n_{0}
\end{gathered}
$$

where $W(x)$ denotes the solution of the equation $y^{*} \exp (y)=x$, which is an Lambert Function, i.e. inverse function of $f(w)=w^{*} \exp (w)$. 


\subsection{Optimal contract design under asymmetric information}

Under the asymmetric information scenario, SUs may hide their personal information to the PU. PU cannot know each SU's type, it only knows the knowledge of the set of SUs' types and the number of each type. In this case, a feasible contract must satisfy both the individual rationality constraint in Definition 2 and the incentive compatibiltiy constraint defined as follows.

Definition 3 (Incentive Compatibility, IC). A contract satisfies the IC constraint if for each type- $\theta_{k} \mathrm{SU}$, it preferes to choose the contract item for $\theta_{k}$, i.e.,

$$
\theta_{k} b_{k}-p_{k} \geq \theta_{k} b_{j}-p_{j} \quad \forall k, j \in \Omega
$$

IC constraint can ensure that SU with type- $\theta_{k}$ will achieve its maximum payoff by choosing the $k$ th contract item.

Then, the optimization problem of PU can be written as:

$$
\max _{\left\{\left(p_{k}, b_{k}\right), \forall k \in \Omega\right\}} U_{P U}\left(\left(p_{k}, b_{k}\right), k \in \Omega\right)
$$

subject to

$$
\left\{\begin{array}{l}
\left(p_{k}, b_{k}\right) \geq 0, \quad \forall k \in \Omega \\
\theta_{k} b_{k}-p_{k} \geq 0, \quad \forall k \in \Omega \\
\theta_{k} b_{k}-p_{k} \geq \theta_{k} b_{j}-p_{j} \quad \forall k, j \in \Omega \\
\frac{1}{2} R_{d}+\frac{1}{2}\left(1-\sum_{k \in \Omega} b_{k}\right) \log \left(1+\frac{\sum_{k \in N} p_{k}}{n_{0}}\right) \geq R_{d}
\end{array}\right.
$$

Next, we show that a feasible contract should satisfy the following conditions.

Proposition 1. For any feasible contract $p_{i} \geq p_{j}$ if and only if $b_{i} \geq b_{j} \forall i, j \in \Omega$.

Proof. The proof can be divided into the following two parts.

Firstly, we prove the suffient of proposition 1. According to the IC constraint, we have $\theta_{i} b_{i}-p_{i} \geq \theta_{i} b_{j}-p_{j}$ i.e., $\theta_{i}\left(b_{i}-b_{j}\right) \geq p_{i}-p_{j}$. Due to $p_{i} \geq p_{j}$, we can obtain $\theta_{i}\left(b_{i}-b_{j}\right) \geq p_{i}-p_{j}>0$ and thus $b_{i} \geq b_{j}$.

Next, we proof the necessity. To satisfy the IC constraint, we have $\theta_{j} b_{j}-p_{j} \geq \theta_{j} b_{i}-p_{i}$, i.e., $p_{i}-p_{j} \geq \theta_{j} b_{i}-\theta_{j} b_{j}=\theta_{j}\left(b_{i}-b_{j}\right)$. Due to $b_{i} \geq b_{j}$, we can obtain $p_{i}-p_{j} \geq \theta_{j}\left(b_{i}-b_{j}\right)>0$ and thus $p_{i} \geq p_{j}$.

Proposition 1 shows that the SU who contributes more power at PU's receiver should get more bandwidth allocation, and vice versa.

The following Proposition 2 shows the second necessary condition for contract feasibility.

Proposition 2. For any feasible contract, if $\theta_{i}>\theta_{j}$, then $b_{i}>b_{j}, \forall i, j \in \Omega$.

Proof. We prove it by using the contradiction. Assume there is such a situation $b_{i}<b_{j}$ with $\theta_{i}>\theta_{j}$. Then we have $\theta_{i} b_{j}+\theta_{j} b_{i}>\theta_{i} b_{i}+\theta_{j} b_{j}$. While the feasible contract items for type- $\theta_{i}$ and type- $\theta_{j}$ need to satisfy the IC constraint, i.e., $\theta_{i} b_{i}-p_{i} \geq \theta_{i} b_{j}-p_{j}$ and $\theta_{j} b_{j}-p_{j} \geq \theta_{j} b_{i}-p_{i}$. Combining the above two inequalities, we have $\theta_{i} b_{i}+\theta_{j} b_{j} \geq \theta_{i} b_{j}+\theta_{j} b_{i}$, which is in contradiction with the former assumption, and thus complete the proof. 
Proposition 2 shows that compared with the low type SU, higher type SU should be allocated more transmission bandwidth. Combined with Proposition 1, we can find that all feasible power-bandwidth combination items should satisfy $0 \leq p_{1} \leq p_{2} \leq \ldots \leq p_{K}$, $0 \leq b_{1} \leq b_{2} \leq \ldots \leq b_{K}$.

As the second derivation of $U_{P U}$ is always negative, we can find that (25) is a nonconvex problem, which is difficult to solve directly. We obtain the optimal power and bandwidth allocation with the following two steps. Firstly, we obtain the optimal power allocation with fixed feasible bandwidth allocations. Then drive the optimal bandwidth allocation. We will show in the numerical results that there is no performance gap between the above solution with the exhaustive search.

Proposition 3. With fixed bandwidth allocations, the optimal unique relay powers satisfy

$$
\begin{aligned}
& p_{1}^{*}\left(\left\{b_{k}, \forall k\right\}\right)=\theta_{1} b_{1} \\
& p_{k}^{*}\left(\left\{b_{k}, \forall k\right\}\right)=\theta_{1} b_{1}+\sum_{i=2}^{k} \theta_{i}\left(b_{i}-b_{i-1}\right)
\end{aligned}
$$

Proof. PU wants the involved SUs to forward its signal with as high power as possible when SUs' IR and IC constraints are satisfied. According to IR constraint, $p_{1}$ equals to $\theta_{1} b_{1}$. Due to type- $\theta_{k}$ SU's IC constraint, we have $\theta_{k} b_{k}-p_{k} \geq \theta_{k} b_{j}-p_{j}, k \neq j$. For any $k=2,3, \ldots, K$, we can obtain $p_{k-1}+\theta_{k-1}\left(b_{k}-b_{k-1}\right) \leq p_{k} \leq p_{k-1}+\theta_{k}\left(b_{k}-b_{k-1}\right)$. Thus, $p_{k}$ equals to $p_{k-1}+\theta_{k}\left(b_{k}-b_{k-1}\right)$ for $2 \leq k \leq K$.

Next we prove that the above contract items satisfy the IR and IC constraints. It is easy to find that the contract items in Proposition 3 meet the IR constraint. We now prove that they satisfy IC constraint, i.e.,

$$
\theta_{k} b_{k}-p_{k} \geq \theta_{k} b_{j}-p_{j}, k \neq j
$$

We have $p_{j}-p_{k}=\sum_{i=2}^{j} \theta_{i}\left(b_{i}-b_{i-1}\right)-\sum_{i=2}^{k} \theta_{i}\left(b_{i}-b_{i-1}\right)$. Combining with Proposition 1 and Proposition 2, we can obtain

$$
\begin{aligned}
p_{j}-p_{k} & =\sum_{i=k+1}^{j} \theta_{i}\left(b_{i}-b_{i-1}\right) \\
& \geq \theta_{k+1}\left(b_{k+1}-b_{k}+b_{k+2}-b_{k+1}+\ldots+b_{j}-b_{j-1}\right) \quad j \geq k \\
& =\theta_{k+1}\left(b_{j}-b_{k}\right) \geq \theta_{k}\left(b_{j}-b_{k}\right) \\
p_{j}-p_{k} & =-\left(\sum_{i=j+1}^{k} \theta_{i}\left(b_{i}-b_{i-1}\right)\right) \\
\geq & -\left(\theta_{k}\left(b_{j+1}-b_{j}+b_{j+2}-b_{j+1}+\ldots+b_{k}-b_{k-1}\right)\right) \quad j<k \\
& =-\left(\theta_{k}\left(b_{k}-b_{j}\right)\right)=\theta_{k}\left(b_{j}-b_{k}\right)
\end{aligned}
$$

Thus, (25) is proved.

Using proposition 3, PU's utility maximization problem can be simplify as

$$
\max _{\left\{b_{k}, \forall k \in \Omega\right\}} \frac{1}{2} R_{d}+\frac{1}{2}\left(1-\sum_{k \in \Omega} b_{k}\right) \log \left(1+\frac{\theta_{1} b_{1}+\sum_{k=2}^{K}\left(\theta_{1} b_{1}+\sum_{i=2}^{k} \theta_{i}\left(b_{i}-b_{i-1}\right)\right)}{n_{0}}\right)
$$

subject to 


$$
\left\{\begin{array}{l}
b_{1} \leq b_{2} \leq \ldots \leq b_{K} \\
\frac{1}{2} R_{d}+\frac{1}{2}\left(1-\sum_{k \in \Omega} b_{k}\right) \log \left(1+\frac{\theta_{1} b_{1}+\sum_{k=2}^{K}\left(\theta_{1} b_{1}+\sum_{i=2}^{k} \theta_{i}\left(b_{i}-b_{i-1}\right)\right)}{n_{0}}\right) \geq R_{d}
\end{array}\right.
$$

It can be further simplified with theorem 2 as follows.

Theorem 2. In the optimal contract, only the contract item with the highest type is positive and all the other contract items are zero, i.e., $\left(p_{K}, b_{K}\right)>0$ and $\left(p_{k}, b_{k}\right)=0, \forall k<K$.

Theorem 2 is easy to be proved with the similar method as theorem 1.

Using Theorem 2, (30) can be further simplified as:

$$
\max _{\left(b_{K} \geq 0\right)} \frac{1}{2} R_{d}+\frac{1}{2}\left(1-b_{K}\right) \log \left(1+\frac{\theta_{K} b_{K}}{n_{0}}\right)
$$

Notice that (31) under asymmetric information is the same as the (19) under symmetric information. The optimal contract items under two scenarios are the same in our model. Then, the optimal bandwidth allocation can be obtained by using the same methdod as in the symmetric information.

\section{Numerical results and Discussions}

The performance of the proposed scheme is shown in this section. Since the optimal contract in the case of symmetric information and asymmetric information are the same, we do not distinguish them in the following simulation results. Considering a typical cooperative system composed of one PU and three SUs. Without loss of generality, we assume that the noise for all the links is white Gaussian noise.

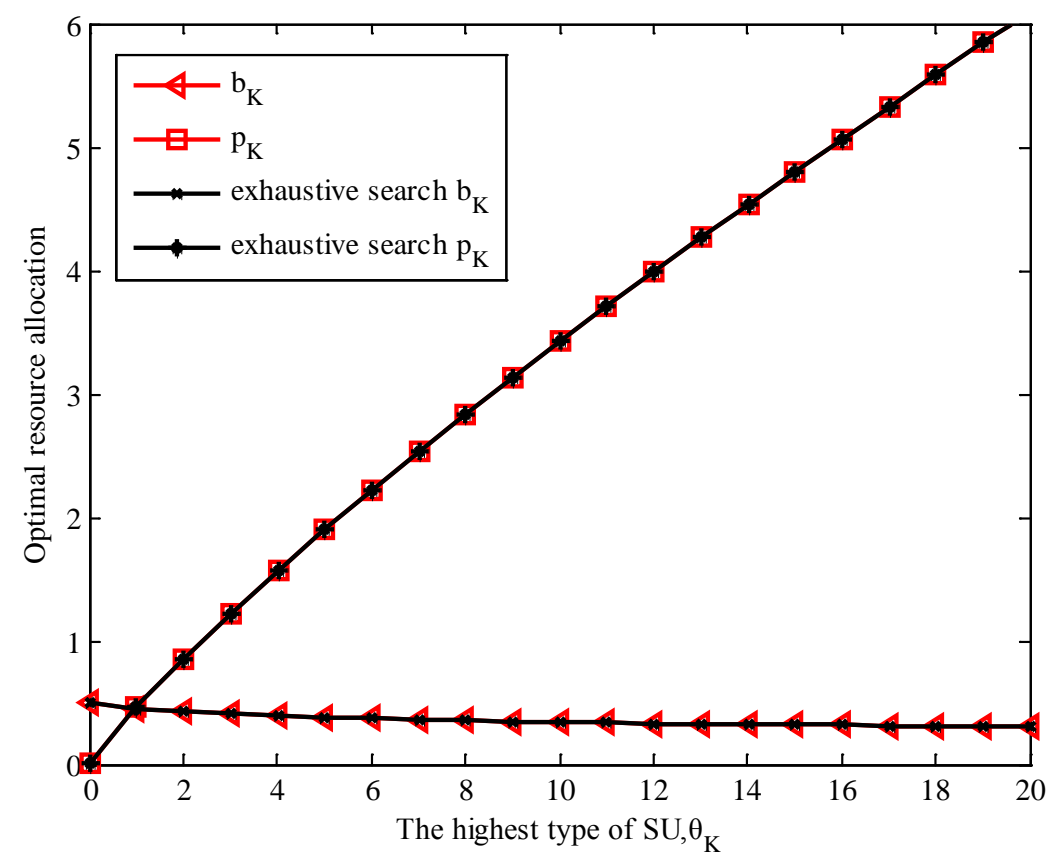

Fig. 3. Optimal bandwidth and power allocation vs. the highest SU type 
Fig. 3 shows the optimal bandwidth and power allocations versus the highest SU's type $\theta_{K}$. We can observe from Fig. 3 that the optimal bandwidth decreases monotonically with the highest type of SU, while the optimal power increases. The SU with larger $\theta_{K}$ offers higher received power at PR. When the highest type of SU becomes large, PU only needs to allocate a small portion of bandwidth to SU to obtain the relay power. This is the reason why PU prefers to choose a high type SU. As shown in Fig. 3, there is no performance gap between our proposed joint power and bandwidth allocation and the exhaustive search.

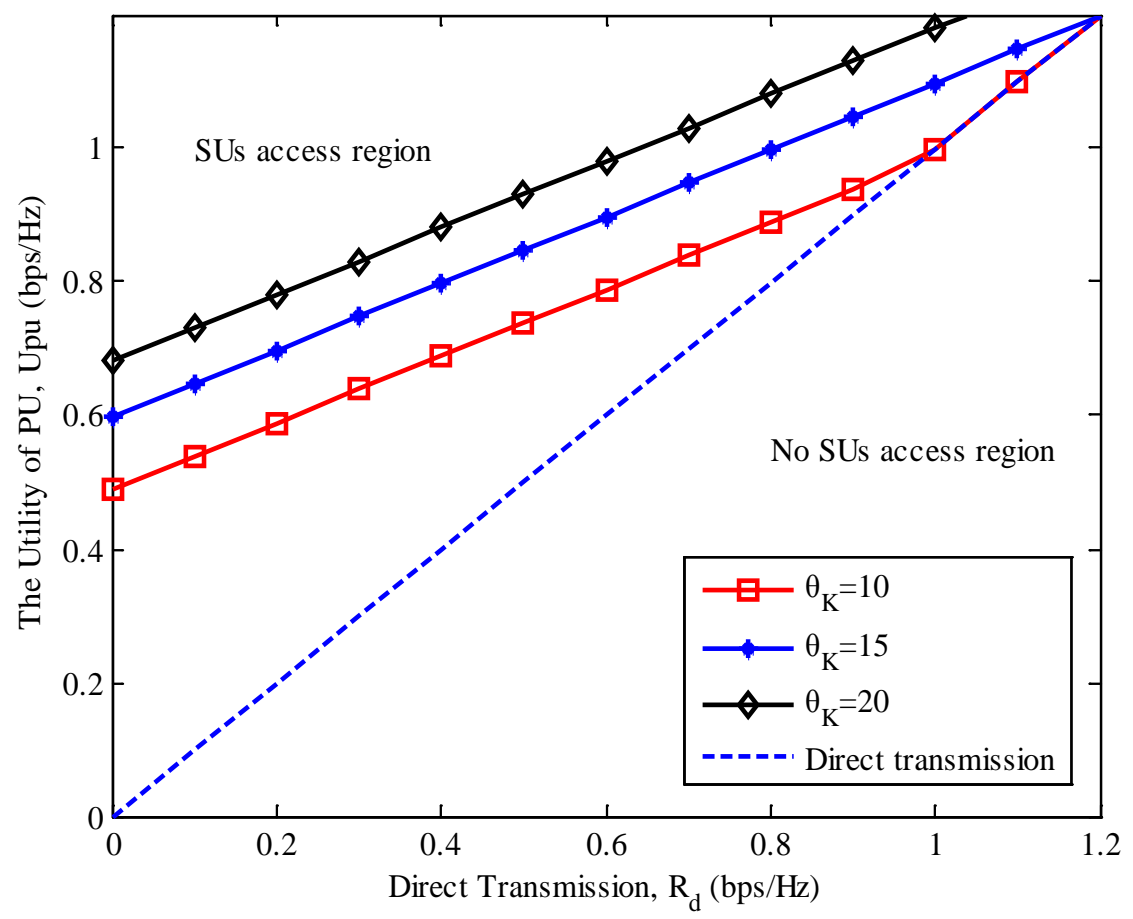

Fig. 4. The utility of PU vs. direct transmission with different $\theta_{K}$

Fig. 4 shows the PU's utility versus direct transmission rate $R_{d}$ with different highest type- $\theta_{K}$. From Fig. 4 we can find that PU's utility increases with $R_{d}$ and $\theta_{K}$. With higher types, SUs will gain more opportunities to access PU's spectrum. The dotted line indicates the transmission rate of the PU when there is no SU operating. It divides the plane into two regions, which are SUs access region and no SUs access region. When $\theta_{K}=10$ and $R_{d}>1$, the PU's utility of cooperation will be smaller than its direct transmission rate. Then, SUs will not be allowed to access to the PU's spectrum. Therefore, the PU's utility equals to the utility of direct transmission. The incentive for the PU to share the spectrum with SUs increases as the type- $\theta_{K}$ increases. It is because of PU's performance constraint, the PU will not choose SUs to forward signals when $R_{d}$ is large enough, which results no SUs can access to the PU's spectrum.

Fig. 5 shows SU's rate versus SU's highest type- $\theta_{K}$ with different received power $p_{s}$. We can find that the SU have the opportunity to transmit its own signal although its utility is zero. The SU with better channel condition will get higher rate during the cooperation with the same transmission power. SU's rate decreases when $\theta_{K}$ becomes large. This is because that the 
accessing bandwidth decreases with the highest type of SU as shown in Fig. 3.

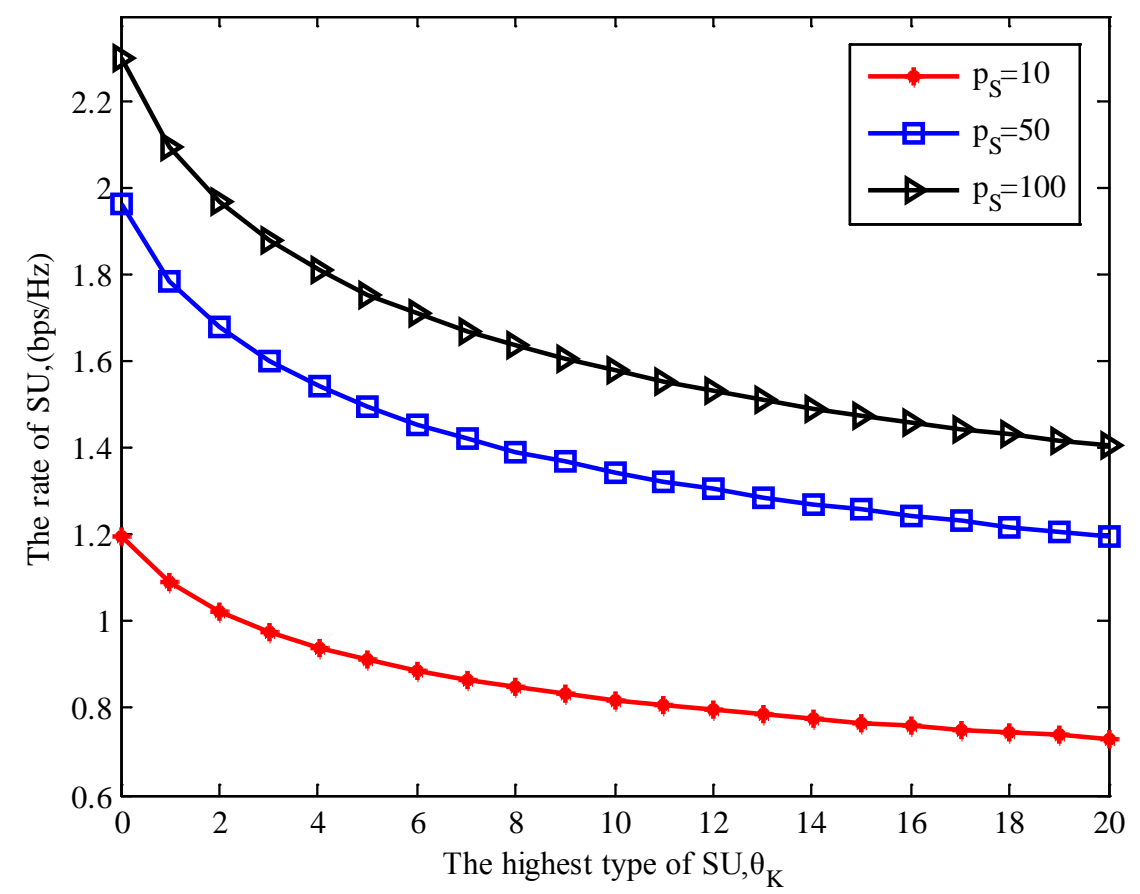

Fig. 5. The rate of SU vs. the highest SU's type

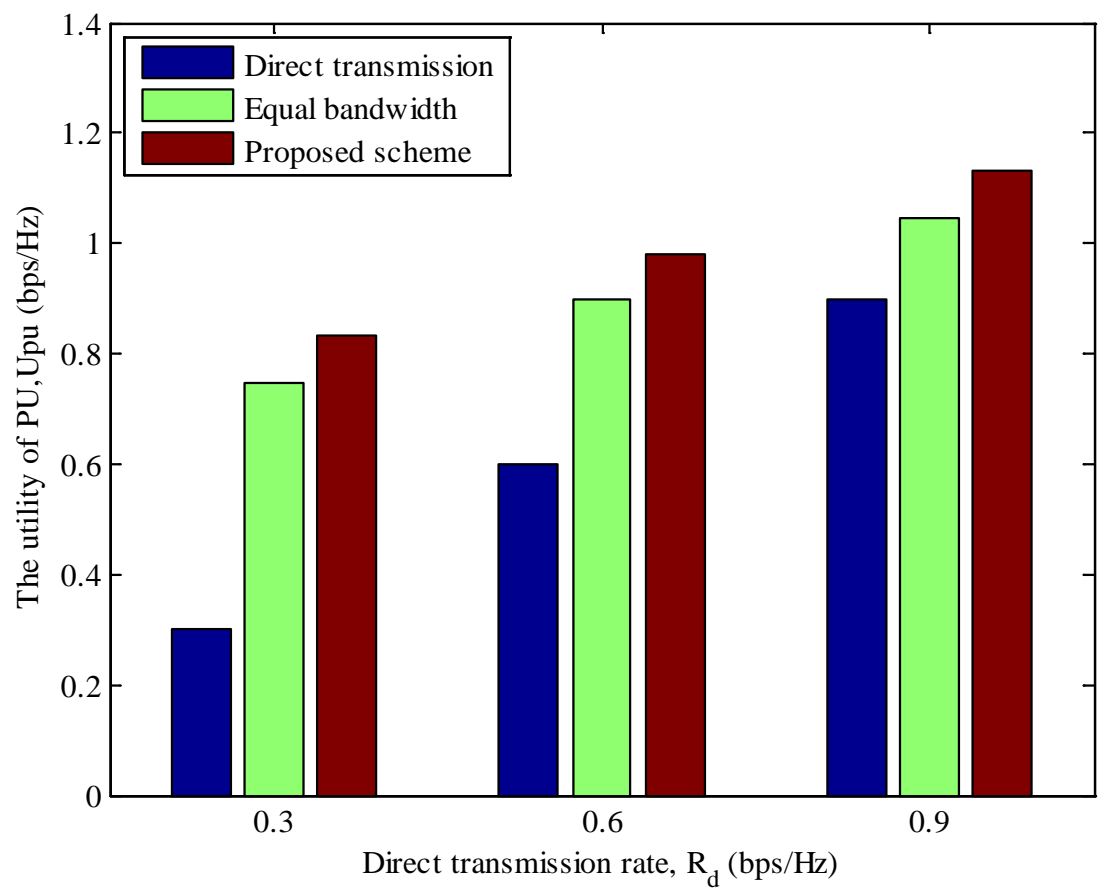

Fig. 6. The utility of PU under different scheme 
Fig. 6 compares PU's utility with our method, direct transmission method and the equal bandwidth allocation method. In the equal bandwidth allocation method, all selected SUs are allocated with the same access bandwidth. From Fig. 6, we can find that PU can only obtain a small utility when it transmits signal directly without any SUs access. Fig. 6 shows that our proposed method outperforms the other two benchmark methods. It is because that with highest type, SU can offer the largest help to the PU's utility under the condition of a given total available bandwidth. In addition, PU's willingness to cooperate decreases with the increase of direct transmission rate.

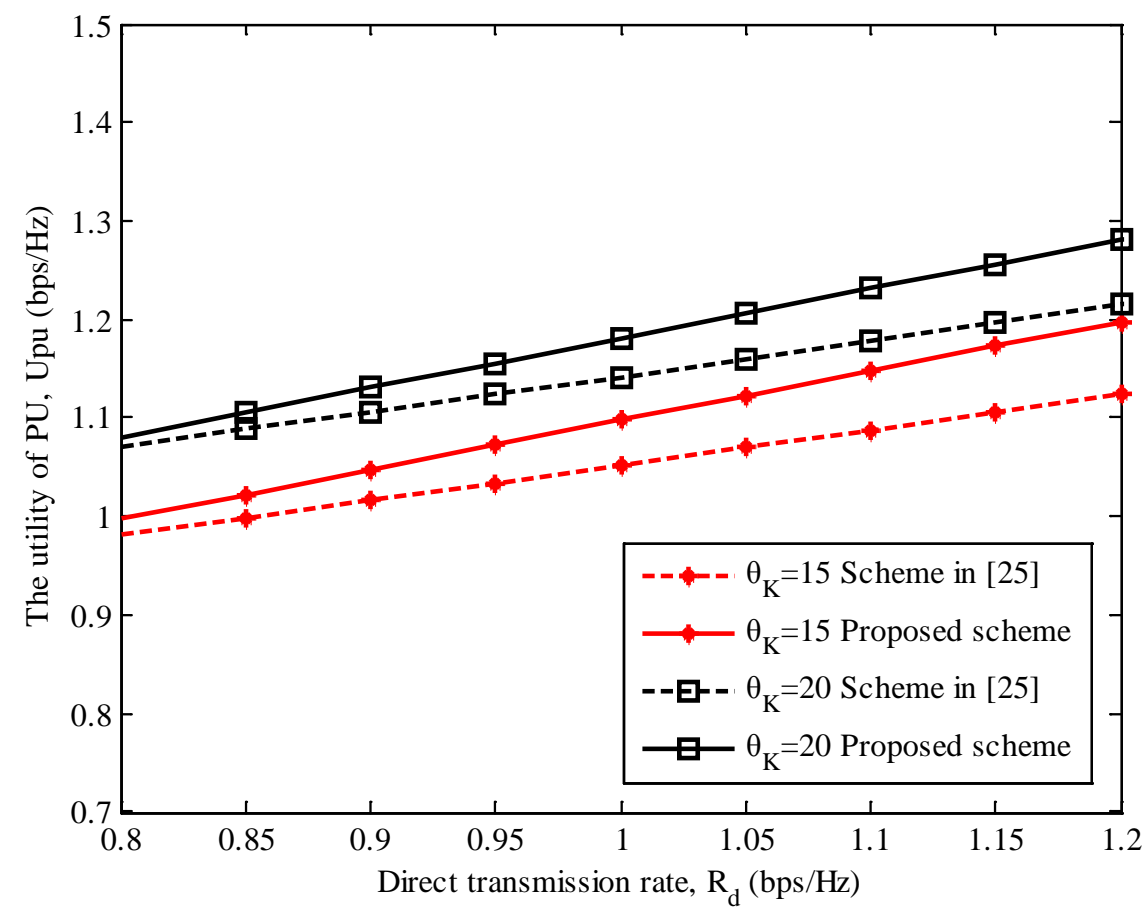

Fig. 7. The utility of PU comparison with different schemes

Fig. 7 illustrates PU's utility with our proposed scheme and the spectrum sharing scheme proposed in [25]. The contract design scheme in [25] includes the combination of relaying power and spectrum accessing time. As shown in Fig. 7, we can find that PU's utility increases with $R_{d}$ and the highest type of SU $\theta_{K}$. We can observe that PU obtains higher utility with proposed scheme when the highest type of SU is given. Thus, the allocation of resource is more reasonable in the proposed method.

\section{Conclusion}

In this paper, we proposed a contract theory based cooperative spectrum sharing scheme with joint power and bandwidth optimization under asymmetric information. The PU hires proper SUs as cooperative relay to forward its signal and the involved SUs obtain opportunities to access licensed spectrum. Each SU chooses the optimal contract item according to its personal information. The proposed scheme takes into account both PU's and SUs' benefits. We study the design of optimal contract under two different scenarios and derive the optimal accessing bandwidth and relaying power, which can maximum PU's utility. 
Numerical results are presented to show PU’s significantly improved performance.

\section{References}

[1] T. Yucek and H. Arslan, "A survey of spectrum sensing algorithms for cognitive radio applications,” IEEE Communications Surveys \& Tutorials, vol. 11, no. 1, pp. 116-130, 2009. Article (CrossRef Link).

[2] G. Ding, J. Wang, Q. Wu, L. Zhang, Y. Zou and Y. Yao, "Robust Spectrum Sensing with Crowd Sensors,” IEEE Transactions on Communications, vol. 62, no. 9, pp. 3129-3143, September. 2014. Article (CrossRef Link).

[3] G. Ding, Q. Wu, Y. Yao, J. Wang and Y. Chen, "Kernel-based learning for statistical signal processing in cognitive radio networks: Theoretical foundations, example applications, and future directions,” IEEE Signal Processing Magazine, vol. 30, no. 4, pp. 126-136, July. 2013.

Article (CrossRef Link).

[4] J. Liu, H. Ding, Y. Cai, H. Yue, Y. Fang and S. Chen, “An Energy-Efficient Strategy for Secondary Users in Cooperative Cognitive Radio Networks for Green Communications,” IEEE Journal on Selected Areas in Communications, vol. 34, no. 12, pp. 3195-3207, December 2016. Article (CrossRef Link).

[5] W. Yang and X. Zhao, "Robust resource allocation for orthogonal frequency division multiplexing-based cooperative cognitive radio networks with imperfect channel state information,” IET Communications, vol. 11, no. 2, pp. 273-281, January 2017. Article (CrossRef Link).

[6] Y. Han, A. Pandharipande and S. H. Ting, "Cooperative spectrum sharing via controlled amplify-and-forward relaying," in Proc. of International Symposium on Personal, Indoor and Mobile Radio Communications, pp. 1-5, September 2008. Article (CrossRef Link).

[7] Songze Li, U. Mitra, V. Ratnam and A. Pandharipande, "Jointly cooperative decode-and-forward relaying for secondary spectrum access,” in Proc. of Annual Conference on Information Sciences and Systems (CISS), pp. 1-6, March 2012. Article (CrossRef Link).

[8] H. Y. Gao, W. Ejaz and M. Jo, "Cooperative Wireless Energy Harvesting and Spectrum Sharing in 5G Networks,” IEEE Access, vol. 4, pp. 3647-3658, 2016. Article (CrossRef Link).

[9] N. U. Hasan, W. Ejaz, N. Ejaz, H. S. Kim, A. Anpalagan and M. Jo, "Network Selection and Channel Allocation for Spectrum Sharing in 5G Heterogeneous Networks," IEEE Access, vol. 4, pp. 980-992, 2016. Article (CrossRef Link).

[10] Y. Zou, Y. D. Yao and B. Zheng, "Cognitive Transmissions with Multiple Relays in Cognitive Radio Networks,” IEEE Transactions on Wireless Communications, vol. 10, no. 2, pp. 648-659, February 2011. Article (CrossRef Link).

[11] Y. Zou, Y. D. Yao and B. Zheng, "Cooperative relay techniques for cognitive radio systems: Spectrum sensing and secondary user transmissions," IEEE Communications Magazine, vol. 50, no. 4, pp. 98-103, April 2012. Article (CrossRef Link).

[12] B. Nazari and A. Jamalipour, "Cooperative communication with asymmetric channel state information: A contract theoretic modeling approach,” China Communications, vol. 10, no. 1, pp. 31-43, January 2013. Article (CrossRef Link).

[13] H. Wang, L. Gao, X. Gan, X. Wang and E. Hossain, "Cooperative Spectrum Sharing in Cognitive Radio Networks: A Game-Theoretic Approach,” in 2010 IEEE International Conference on Communications, pp. 1-5, May 2010. Article (CrossRef Link).

[14] M. Tao and Y. Liu, "Spectrum leasing and cooperative resource allocation in cognitive OFDMA networks,” Journal of Communications and Networks, vol. 15, no. 1, pp. 102-110, Febryary 2013. Article (CrossRef Link).

[15] Y. Wu and W. Z. Song, "Cooperative Resource Sharing and Pricing for Proactive Dynamic Spectrum Access via Nash Bargaining Solution," IEEE Transactions on Parallel and Distributed Systems, vol. 25, no. 11, pp. 2804-2817, November 2014. Article (CrossRef Link). 
[16]F. Wang, M. Krunz and S. Cui, "Price-Based Spectrum Management in Cognitive Radio Networks," in Proc. of International Conference on Cognitive Radio Oriented Wireless Networks and Communications, pp. 70-78, August 2007. Article (CrossRef Link).

[17] X. Wang, K. Ma, Q. Han, Z. Liu and X. Guan, "Pricing-based spectrum leasing in cognitive radio networks,” IET Networks, vol. 1, no. 3, pp. 116-125, September 2012. Article (CrossRef Link).

[18] L. Gao, Y. Xu and X. Wang, "MAP: Multiauctioneer Progressive Auction for Dynamic Spectrum Access,” IEEE Transactions on Mobile Computing, vol. 10, no. 8, pp. 1144-1161, August 2011. Article (CrossRef Link).

[19] M. Pan, J. Sun and Y. Fang, "Purging the Back-Room Dealing: Secure Spectrum Auction Leveraging Paillier Cryptosystem," IEEE Journal on Selected Areas in Communications, vol. 29, no. 4, pp. 866-876, April 2011. Article (CrossRef Link).

[20] Q. Shi, C. Comaniciu and K. Jaffres-Runser, "An Auction-Based Mechanism for Cooperative Sensing in Cognitive Networks," IEEE Transactions on Wireless Communications, vol. 12, no. 8, pp. 3649-3661, August 2013. Article (CrossRef Link).

[21]D. Yang, X. Fang and G. Xue, "Game theory in cooperative communications," IEEE Wireless Communications, vol. 19, no. 2, pp. 44-49, April 2012. Article (CrossRef Link).

[22] Y. Zhang, M. Pan, L. Song, Z. Dawy and Z. Han, "A Survey of Contract Theory-Based Incentive Mechanism Design in Wireless Networks," IEEE Wireless Communications, vol. 24, no. 3, pp. 80-85, 2017. Article (CrossRef Link).

[23] L. Gao, X. Wang, Y. Xu and Q. Zhang, "Spectrum Trading in Cognitive Radio Networks: A Contract-Theoretic Modeling Approach," IEEE Journal on Selected Areas in Communications, vol. 29, no. 4, pp. 843-855, April 2011. Article (CrossRef Link).

[24] B. Nazari and A. Jamalipour, "Contract-based cooperative spectrum sharing in cognitive radio networks," in Proc. of IEEE Global Communications Conference, pp. 888-892, December 2014. Article (CrossRef Link).

[25] L. Duan, L. Gao and J. Huang, "Cooperative Spectrum Sharing: A Contract-Based Approach," IEEE Transactions on Mobile Computing, vol. 13, no. 1, pp. 174-187, January 2014. Article (CrossRef Link).

[26] J. N. Laneman and G. W. Wornell, "Distributed Space-Time-Coded Protocols for Exploiting Cooperative Diversity in Wireless Networks," IEEE Trans. Information Theory, vol. 49, no. 10, pp. 2415-2425, October. 2003. Article (CrossRef Link). 

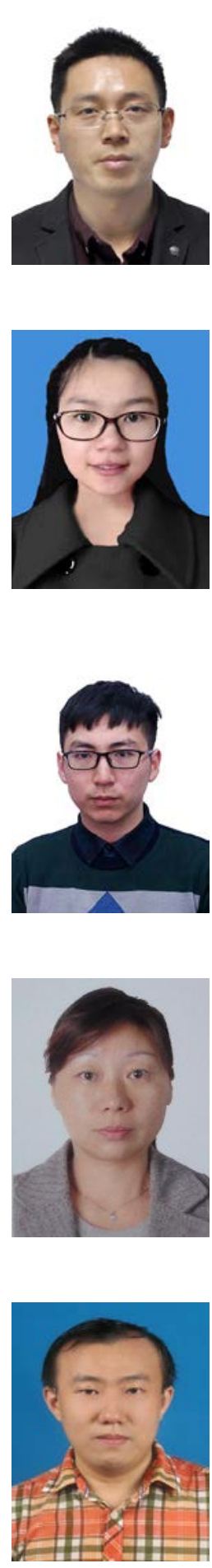

Lu Weidang received the Ph.D. degree in Information and Communication Engineering from Harbin Institute of Technology. He is currently an Associate Professor with the College of Information Engineering, Zhejiang University of Technology, Hangzhou, China. He was a visitor at Nanyang Technological University, The Chinese University of HongKong and Southern University of Science and Technology. His current research interests include simultaneous wireless information and power transfer, cooperative communications, OFDM and cognitive radio.

He Chenxin is currently a Master candidate at Zhejiang University of Technology, Hangzhou, China. Her current research interests include cognitive radio, cooperative communications.

Lin Yuanrong is currently a Master candidate at Zhejiang University of Technology, Hangzhou, China. Her current research interests include cognitive radio, simultaneous wireless information and power transfer.

Peng Hong is an Associate Professor with Zhejiang University of Technology, China. Her research interests include network optimization and cognitive radio.

Liu Xin received the Ph.D. degree in Information and Communication Engineering from Harbin Institute of Technology. He is now with Dalian University of Technology as an Associate Professor. He was a research fellow of Nanyang Technology University. His current research interests include cooperative communications and cognitive radio. 